\title{
Ansiedade cardíaca: uma análise em ambulatórios cardiológicos subespecializados
}

\section{Cardiac anxiety: an analysis in subspecialized cardiac outpatient}

Patrícia Helena Alves Maciel ${ }^{1}$. Ricardo Pereira Silva². Carlos Roberto Martins Rodrigues Sobrinho ${ }^{3}$. Antônio Reis de Sá Junior ${ }^{4}$. Leticia Marinho Pontes ${ }^{5}$. Brena Ferreira dos Santos ${ }^{5}$.

1 Médica Residente de Cardiologia, Hospital Universitário Walter Cantídio (HUWC), Universidade Federal do Ceará (UFC), Fortaleza, Ceará, Brasil. 2 Professor de Cardiologia, Faculdade de Medicina, Universidade Federal do Ceará (UFC), Fortaleza, Ceará, Brasil. 3 Serviço de Cardiologia, Hospital Universitário Walter Cantídio (HUWC), Professor, Universidade Federal do Ceará (UFC), Fortaleza, Ceará, Brasil. 4 Médico Psiquiatra, Professor do Departamento de Medicina e Enfermagem, Universidade Federal de Viçosa (UFV), Viçosa, Minas Gerais, Brasil. 5 Estudante de Medicina, Faculdade de Medicina, Universidade Federal do Ceará (UFC), Fortaleza, Ceará, Brasil.

\section{RESUMO}

Objetivos: a condição clínica ansiedade cardíaca é o medo de sensações relacionadas ao coração por consequências negativas dos eventos cardíacos. O Questionário de Ansiedade Cardíaca (QAC) é uma ferramenta internacionalmente utilizada no rastreamento dessa ansiedade. Este artigo objetiva verificar as propriedades psicométricas da versão brasileira do instrumento QAC. Metodologia: o QAC foi aplicado em amostra de 239 pacientes distribuídos entre cinco ambulatórios de cardiologia (arritmia, hipertensão, miocardiopatia, doença coronariana e valvulopatia) para avaliação da confiabilidade e extração fatorial da amostra total e para a Análise Fatorial Multigrupo entre esses ambulatórios. Resultados: os resultados indicam que o QAC apresenta boa consistência interna (Alfa de Cronbach $=0,79$ ) e quando da realização da análise fatorial, o modelo com quatro fatores mostrou ser o mais adequado, com uma explicação de $54,9 \%$ da variância total no geral. A estrutura fatorial do QAC apresentou a mesma importância para o construto de ansiedade cardíaca, independente do ambulatório. Ao comparar as médias fatoriais entre os ambulatórios, houve diferenças significativas apenas no fator evitação. Conclusão: Os resultados comprovam a utilidade da escala QAC para uso no contexto ambulatorial brasileiro, sendo fundamental para o desenvolvimento de estratégias de rastreio da ansiedade cardíaca, bem como para propostas de intervenções baseadas nessas medidas.

Palavras-chave: Ansiedade. Psicometria. Instituições de assistência ambulatorial. Cardiologia.

\section{ABSTRACT}

Objectives: The clinical condition heart anxiety is the fear of feelings related to the heart by negative consequences of cardiac events. The Heart Anxiety Questionnaire (CAQ) is an internationally used tool to screening this anxiety. This article aims to verify psychometric properties of the Brazilian version of the CAQ instrument. Methodology: The CAQ was applied in a sample of 239 patients distributed among five cardiology clinics (arrhythmia, hypertension, cardiomyopathy, coronary disease and valvulopathy) in order to evaluate the reliability and factorial extraction of the total sample and to the Multigroup Factor Analysis among these outpatient clinics. Results: The results indicate that CAQ shows good internal consistency (Cronbach's alpha $=0.79$ ) and after factorial analysis, the 4 -factor model was found to be the most adequate, explaining $54.9 \%$ of the total variance in general. The CAQ factorial structure showed the same relevance for the construct cardiac anxiety, and that was regardless the clinic. The comparison between factorial means among clinics showed significant differences only in the avoidance factor. Conclusion: The results confirm the usefulness of the CAQ scale in the Brazilian outpatient, being fundamental for the development of strategies for screening for heart anxiety, as well as for proposals for interventions based on these measures.

Keywords: Anxiety. Psychometrics. Ambulatory care facilities. Cardiology.

Autor correspondente: Patrícia Helena Alves Maciel, Rua Eduardo Salgado, 303, Aldeota, Fortaleza, Ceará. CEP: 60150-140. Telefone: +55 85 99969-8340. E-mail: patriciahellena@yahoo.com.br

Conflito de interesses: Não há qualquer conflito de interesses por parte de qualquer um dos autores.

Recebido em: 29 Nov 2017; Revisado em: 15 Mai 2018; Aceito em: 15 Mai 2018. 


\section{INTRODUÇÃO}

O conceito de ansiedade cardíaca deriva da descrição de cardiofobia. ${ }^{1} \mathrm{O}$ termo cardiofobia foi originalmente utilizado para se referir a pacientes com dor torácica sem uma causa orgânica, associada ao medo de morrer de um ataque cardíaco. ${ }^{2} \mathrm{O}$ estudo seminal sobre cardiofobia foi feito com grupos de pacientes encaminhados para o ambulatório de cardiologia com e sem doença coronariana e que ao serem comparados com voluntários sadios, observou-se que o grupo de pacientes sem doença coronariana apresentava sintomas como dor no peito ou palpitações, acompanhados de um medo excessivo de um evento cardíaco fatal. ${ }^{3} \mathrm{O}$ termo cardiofobia foi então substituído por ansiedade cardíaca que por sua vez é conceitualmente distinta de outros transtornos de ansiedade. ${ }^{3,4} \mathrm{~A}$ ansiedade cardíaca é o medo de sensações e estímulos relacionados ao coração por causa das possíveis consequências negativas dos eventos cardíacos. ${ }^{4}$

Embora já tenha sido estudada em fumantes e também em relação ao status socioeconômico, a maioria dos estudos sobre ansiedade cardíaca tem sido direcionada à sua identificação em pacientes com doença cardíaca. ${ }^{5-12} \mathrm{~A}$ ansiedade cardíaca está relacionada a uma diminuição do funcionamento social e ocupacional, bem como da qualidade de vida. ${ }^{9}$ Infelizmente, com uma preocupante frequência, a ansiedade cardíaca não é reconhecida como um fator que contribui no desfecho de uma condição médica, particularmente nos ambulatórios de cardiologia. ${ }^{12,13}$

O Questionário da Ansiedade Cardíaca (QAC) foi desenvolvido para facilitar a identificação da ansiedade cardíaca. ${ }^{14}$ É um questionário de aplicação rápida, respondido através do autopreenchimento pelos pacientes. No Brasil, o QAC foi traduzido e validado para o português. ${ }^{15,16}$

O objetivo geral deste estudo é verificar as propriedades psicométricas do instrumento QAC em uma amostra que inclui pacientes com doença coronariana, mas também arritmia e outras cardiopatias. Especificamente, busca-se avaliar a consistência interna da escala e analisar de forma exploratória e confirmatória a estrutura fatorial da escala nas amostras de cinco ambulatórios subespecializados de cardiologia.

\section{MATERIAL E MÉTODOS}

O projeto foi aprovado pelo Comitê de Ética em Pesquisa do Hospital Universitário Walter Cantídio (HUWC) pelo CAAE 19861513.5.0000.5045. Foram respeitados todos os princípios éticos para pesquisa com seres humanos, segundo as normas do Conselho Nacional de Saúde e está de acordo com os critérios estabelecidos pela Declaração de Helsinki e suas modificações. O Termo de Consentimento Livre e Esclarecido (TCLE) foi assinado por todos os participantes após os devidos esclarecimentos e antes da coleta de dados. Os dados foram coletados de janeiro a dezembro de 2015.

\section{1) Amostra}

O ambulatório de cardiologia do HUWC é organizado de acordo com diferentes patologias cardíacas. Todos os pacientes dos cinco ambulatórios especializados de cardiologia do HUWC (arritmia, hipertensão, miocardiopatia, doença coronariana e valvulopatia) foram, em ordem de chegada ao atendimento, convidados a participar do estudo. Um total de 239 pacientes distribuídos entre os cinco ambulatórios concordaram em participar e assinaram o termo de consentimento e em seguida, preencheram o Questionário da Ansiedade Cardíaca (QAC), versão em português com 18 itens. Todos os pacientes que concordaram em participar foram incluídos no estudo. Não se utilizou nenhum critério de exclusão.

\section{2) Questionário da ansiedade cardíaca}

O QAC foi traduzido e validado para o português em 2008 e posteriormente em 2013. ${ }^{15,16}$ A versão original do QAC em inglês foi publicada em 2000 e conta com 18 itens distribuídos em três fatores ou subescalas: medo e preocupação com sensações cardíacas; evitação cardioprotetora de atividades que podem ocasionar sintomas; atenção e monitoramento de estímulos cardíacos. ${ }^{14}$ No entanto, um novo estudo em 2008 propõe uma quarta subescala para analisar a necessidade de tranquilização. ${ }^{10}$ Este termo é traduzido do inglês reassurance-seeking e descreve a necessidade que certos pacientes têm de repetidas vezes checarem com familiares ou com os médicos se está tudo bem com relação à saúde cardíaca, nesse sentido, eles buscam aplacar o medo que sentem com relação aos sintomas. A versão validada do QAC para o português sugere que a estrutura fatorial do QAC pode ter dois, três ou quatro fatores, porém, optou por uma versão do QAC de 14 itens divididos em duas subescalas (medo/ hipervigilância e evitação), portanto, uma solução com dois fatores. ${ }^{16}$ Consequentemente, como encontramos na literatura diferentes modelos fatoriais do QAC, optamos por extrair a melhor estrutura fatorial para a nossa amostra utilizando a versão original do QAC traduzida para o português que contem 18 itens. $^{15}$

\section{3) Análise estatística}

Foram realizadas análises descritivas e de consistência interna por meio do Alfa de Cronbach, considerando os valores totais e com itens excluídos. Visto que um dos nossos objetivos era comparar diferenças entre os ambulatórios ou subgrupos de acordo com os fatores ou subescalas do QAC, para assegurar que as diferenças entre as médias não estariam enviesadas, utilizou-se a Análise Fatorial Confirmatória Multigrupo (AFCMG). ${ }^{17}$ A AFCMG tem sido empregada em estudos de comparação de subgrupos de uma mesma amostra e se faz necessária porque, a não ser que seja rigorosamente testado, não se pode afirmar que a configuração e os parâmetros de determinado instrumento psicométrico são semelhantes em diferentes populações ou subgrupos. Não se encontrou na literatura nenhum trabalho semelhante até a presente data. Os dados foram tabulados no Microsoft Excel $^{\circledR}$, e então exportados para os softwares SPSS versão 21 para Windows e $\mathrm{R}$ versão 3.2.2 (R Core Team, 2015) com o pacote Lavaan 0.5 para a realização das análises estatísticas. 


\section{RESULTADOS}

\section{1) Análise descritiva e de consistência interna}

Para esta análise foram utilizados os dados provenientes da aplicação do CAQ a 239 pacientes, divididos em cinco ambulatórios (ambulatório de arritmia, de DAC - doença aterosclerótica coronariano, de hipertensão, de miocardiopatia e de valvulopatia). $\mathrm{Na}$ análise dos dados distribuídos na forma demonstrada na Tabela 1 encontramos o predomínio do sexo feminino $(59,4 \%)$, a idade dos participantes variando entre 20 e 87 anos e a média do escore total da escala CAQ em 48,66 $(\mathrm{DP}=13,55)$.
Encontrou-se boa consistência interna (Alfa de Cronbach=0,79) da escala para a amostra total. Na Tabela 2 os itens são agrupados por fator e são apresentados os escores individuais de cada item, o desvio padrão (DP), o coeficiente de correlação de item total corrigida, o Alfa de Cronbach, se o item for excluído, e maior carga fatorial de cada item. A média dos escores dos itens variou entre 1,72 para o item 3 e 3,65 para o item 4 . O coeficiente de correlação de item total corrigida apresentou valores entre 0,10 para o item 12 e 0,54 para o item 14 e o Alfa de Cronbach, se o item for excluído, permaneceu ao redor de 0,78 , levando a crer que a exclusão de itens não levou à melhora na confiabilidade da escala e os 18 itens se distribuíram nos quatro fatores com cargas fatoriais variando entre 0,81 com o item 12 no primeiro fator e 0,38 com o item 1 no terceiro fator (Tabela 2).

Tabela 1. Frequência de pacientes por ambulatório especializado de cardiologia. Hospital Universitário Walter Cantídio-UFC, 2015.

\begin{tabular}{lccccc}
\hline Ambulatório & Frequência (\%) & Masculino & Feminino & Idade (DP) & $\begin{array}{c}\text { Escore CAQ } \\
\text { M (DP) }\end{array}$ \\
\hline Arritmia & $55(23,0)$ & 26 & 29 & $59,8(10,6)$ & $52,0(12,7)$ \\
DAC & $63(26,4)$ & 26 & 37 & $63,8(10,1)$ & $49,9(13,1)$ \\
Hipertensão & $49(20,5)$ & 19 & 30 & $62,6(10,4)$ & $46,2(13,4)$ \\
Miocardiopatia & $27(11,3)$ & 12 & 15 & $55,6(12,6)$ & $43,6(14,6)$ \\
Valvulopatia & $45(18,8)$ & 14 & 31 & $54,3(15,2)$ & $48,4(13,9)$ \\
Total & $\mathbf{2 3 9}$ & $\mathbf{9 7}$ & $\mathbf{1 4 2}$ & $\mathbf{5 9 , 9 ( 1 2 , 1 )}$ & $\mathbf{4 8 , 7}(\mathbf{1 3}, \mathbf{6})$ \\
\hline
\end{tabular}

Tabela 2. Média (M), desvio padrão (DP), correlação do item-total, consistência interna (Alfa de Cronbach), se o item for retirado, e carga fatorial dos itens do Questionário da Ansiedade Cardíaca (QAC). Serviço de Cardiologia. Hospital Universitário Walter Cantídio-UFC, 2015.

\begin{tabular}{|c|c|c|c|c|c|}
\hline Item & M & DP & $\begin{array}{l}\text { Correlação } \\
\text { do item-total }\end{array}$ & $\begin{array}{l}\text { Alfa de Cronbach se } \\
\text { o item é retirado }\end{array}$ & $\begin{array}{c}\text { Carga } \\
\text { fatorial }\end{array}$ \\
\hline \multicolumn{6}{|l|}{ Fator 1} \\
\hline 2. Evito esforço físico & 1,77 & 1,14 & 0,31 & 0,78 & 0,7 \\
\hline 5. Pego leve o máximo possível & 1,93 & 1,42 & 0,2 & 0,79 & 0,68 \\
\hline 7. Evito fazer exercícios ou outras atividades físicas & 2,42 & 1,54 & 0,21 & 0,79 & 0,59 \\
\hline 9. Evito atividades que acelerem o meu coração & 1,95 & 1,54 & 0,32 & 0,78 & 0,76 \\
\hline 12. Evito atividades que me façam suar & 1,78 & 1,28 & 0,1 & 0,79 & 0,81 \\
\hline \multicolumn{6}{|l|}{ Fator 2} \\
\hline $\begin{array}{l}\text { 10. Mesmo que os exames estejam normais, eu continuo } \\
\text { me preocupando com o meu coração }\end{array}$ & 2,89 & 1,89 & 0,33 & 0,78 & 0,51 \\
\hline $\begin{array}{l}\text { 14. Quando tenho desconforto no peito ou meu coração } \\
\text { está acelerado, preocupa-me que posso ter um ataque } \\
\text { cardíaco }\end{array}$ & 2,41 & 1,71 & 0,54 & 0,76 & 0,68 \\
\hline $\begin{array}{l}\text { 15. Quando tenho desconforto no peito ou meu coração } \\
\text { está acelerado, tenho dificuldade de me concentrar em } \\
\text { qualquer outra coisa }\end{array}$ & 2,62 & 1,71 & 0,52 & 0,77 & 0,46 \\
\hline
\end{tabular}

Continua. 
Conclusão.

Tabela 2. Média, desvio padrão (DP), correlação do item-total, consistência interna (Alfa de Cronbach), se o item for retirado, e carga fatorial dos itens do Questionário da Ansiedade Cardíaca (QAC). Serviço de Cardiologia. Hospital Universitário Walter Cantídio-UFC, 2015.

\begin{tabular}{|c|c|c|c|c|c|}
\hline Item & $\mathbf{M}$ & DP & $\begin{array}{c}\text { Correlação } \\
\text { do item-total }\end{array}$ & $\begin{array}{l}\text { Alfa de Cronbach se } \\
\text { o item é retirado }\end{array}$ & $\begin{array}{l}\text { Carga } \\
\text { fatorial }\end{array}$ \\
\hline \multicolumn{6}{|l|}{ Fator 2} \\
\hline $\begin{array}{l}\text { 16. Quando tenho desconforto no peito ou meu coração } \\
\text { está acelerado, fico com medo }\end{array}$ & 3,55 & 1,52 & 0,36 & 0,78 & 0,75 \\
\hline \multicolumn{6}{|l|}{ Fator 3} \\
\hline 1. Presto atenção nas batidas do meu coração & 3,62 & 1,66 & 0,4 & 0,77 & 0,38 \\
\hline 6. Verifico minha pulsação & 3,25 & 1,89 & 0,42 & 0,77 & 0,39 \\
\hline $\begin{array}{l}\text { 11. Sinto-me seguro estando próximo a hospitais, } \\
\text { médicos e outros serviços de saúde }\end{array}$ & 3,26 & 1,83 & 0,42 & 0,77 & 0,49 \\
\hline $\begin{array}{l}\text { 17. Quando tenho desconforto no peito ou meu coração } \\
\text { está acelerado, gosto de ser examinado por um médico }\end{array}$ & 2,72 & 1,78 & 0,44 & 0,77 & 0,58 \\
\hline $\begin{array}{l}\text { 18. Quando tenho desconforto no peito ou meu coração } \\
\text { está acelerado, conto para minha família ou amigos }\end{array}$ & 2,85 & 1,61 & 0,21 & 0,79 & 0,6 \\
\hline \multicolumn{6}{|l|}{ Fator 4} \\
\hline 3. Meu coração acelerado me acorda à noite & 1,72 & 1,13 & 0,32 & 0,78 & 0,68 \\
\hline 4. Dor ou desconforto no peito me acordam à noite & 3,65 & 1,63 & 0,42 & 0,77 & 0,63 \\
\hline 8. Posso sentir meu coração no meu peito & 3,43 & 1,8 & 0,48 & 0,77 & 0,44 \\
\hline
\end{tabular}

\section{2) Análise fatorial exploratória e confirmatória}

A análise fatorial exploratória foi feita através do cálculo da medida de adequação da amostra de Kaiser-Meyer-Olkin que gerou um valor significante de 0,784 e do teste de esfericidade de Bartlett (qui-quadrado 1236,687; graus de liberdade 153 e $\mathrm{p}<0,001$ ) demonstrando que a amostra é apropriada para este tipo de análise. A extração fatorial da amostra total foi avaliada com o método de máxima verossimilhança, que possibilita a realização do teste qui-quadrado para avaliar a qualidade de ajustamento do modelo. Além do qui-quadrado, calculou-se também os índices RMSEA (Root Mean Square Error of Approximation) e CFI (Comparative Fit Index) para a seleção de qual modelo, em relação à quantidade de fatores, seria adotado como o mais adequado para esta amostra. De acordo com os critérios utilizados, os valores do qui-quadrado, RMSEA e CFI foram 139,6; 0,055; e 0,929 para o modelo com quatro fatores. Em comparação ao modelo com um (quiquadrado 714,5; RMSEA 0,137; CFI 0,462) e com três fatores (qui-quadrado 227,5; RMSEA 0,064; CFI 0,915), o modelo com quatro fatores apresentou ser o mais adequado, além de apresentar uma explicação de 54,9\% da variância total.

Tendo em vista que um dos nossos objetivos era analisar a predominância de sintomas de uma das quatro subescalas em cada ambulatório, uma questão que se apresentou foi se os itens de um determinado fator apresentavam a mesma importância para diferentes grupos. Para assegurar que a diferença entre as médias não estariam enviesadas, utilizamos a Análise Fatorial
Confirmatória Multigrupo (AFCMG). ${ }^{17}$ A AFCMG tem sido empregada em estudos de comparação de subgrupos de uma mesma amostra. A AFCMG se faz necessária porque, a não ser que seja rigorosamente testada, não podemos afirmar que a configuração e os parâmetros de determinado instrumento psicométrico são semelhantes em diferentes populações ou subgrupos. Os resultados da comparação entre o modelo com cargas livres e o modelo com cargas iguais foram semelhantes entre os nossos ambulatórios (Tabela 3). Neste caso, pode-se aceitar o pressuposto de que a estrutura fatorial (ou as subescalas) do QAC apresenta a mesma importância para o construto de ansiedade cardíaca, independente do ambulatório.

Usando o modelo com cargas iguais para os grupos ambulatoriais, pode-se comparar os escores de um ambulatório com outro (Tabela 4). Ao comparar as médias fatoriais (médias das subescalas) entre os ambulatórios, não houve diferenças significativas em relação aos fatores preocupação, busca por tranquilidade e atenção. As diferenças significativas entre os ambulatórios ocorreram apenas no fator evitação.

Os pacientes do ambulatório de arritmia mostraram mais sintomas evitativos do que os pacientes dos ambulatórios de hipertensão, miocardiopatia e valvulopatia. Os pacientes do ambulatório de doença aterosclerótica coronariana apresentaram mais sintomas evitativos que os pacientes do ambulatório de hipertensão, miocardiopatia e valvulopatia. A diferença no fator evitação entre os pacientes do ambulatório de arritmia e doença coronariana não foi estatisticamente significante. 
Tabela 3. Comparação entre os modelos considerando a invariância métrica e a invariância configural entre os ambulatórios. Serviço de Cardiologia, Hospital Universitário Walter Cantídio-UFC, 2015.

\begin{tabular}{lccccc}
\hline \multicolumn{1}{c}{ Modelos } & Qui-quadrado & G.L & p valor & RMSEA & CFI \\
\hline $\begin{array}{l}\text { Cargas livres para variar entre os } \\
\text { ambulatórios }\end{array}$ & 760,08 & 555 & - & 0,088 & 0,838 \\
$\begin{array}{l}\text { Cargas iguais para os grupos } \\
\text { ambulatoriais }\end{array}$ & 832,14 & 615 & 0,1369 & 0,086 & 0,829 \\
\hline
\end{tabular}

G.L: graus de liberdade.

Tabela 4. Comparação das médias fatoriais entre os ambulatórios. Serviço de Cardiologia, Hospital Universitário Walter Cantídio-UFC, 2015.

\begin{tabular}{lcccc}
\hline Amb 1- Amb 2 & Evitação & Preocupação & Busca por tranquilidade & Atenção \\
\hline Arritmia-DAC & $-0,023$ & $-0,16$ & $-0,17$ & $-0,119$ \\
Arritmia-Hipertensão & $\mathbf{- 0 , 5 4 7}$ & $-0,123$ & 0,002 & $-0,314$ \\
Arritmia-Miocardiopatia & $\mathbf{- 0 , 6 4}$ & $-0,099$ & $-0,324$ & $-0,31$ \\
Arritmia-Valvulopatia & $\mathbf{- 0 , 3 6 1}$ & $-0,095$ & $-0,012$ & $-0,103$ \\
DAC-Hipertensão & $\mathbf{- 0 , 5 2 4}$ & 0,037 & 0,173 & $-0,194$ \\
DAC-Miocardiopatia & $\mathbf{- 0 , 6 1 7}$ & 0,061 & $-0,154$ & $-0,191$ \\
DAC-Valvulopatia & $\mathbf{- 0 , 3 3 8}$ & 0,065 & 0,158 & 0,016 \\
Hipertensão-Miocardiopatia & $-0,093$ & 0,024 & $-0,327$ & 0,003 \\
Hipertensão-Valvulopatia & 0,186 & 0,028 & $-0,015$ & 0,21 \\
Miocardiopatia-Valvulopatia & 0,279 & 0,004 & 0,312 & 0,207 \\
\hline
\end{tabular}

Nota: valores em negrito correspondem a uma diferença significativa $(\mathrm{p}<0,05)$.

\section{DISCUSSÃO}

O presente estudo foi constituído de duas fases complementares com a análise psicométrica dos 18 itens da versão brasileira da escala QAC. Inicialmente foi realizada a análise descritiva e de consistência interna, seguida por análise fatorial exploratória e confirmatória da escala. A análise descritiva mostrou que os ambulatórios avaliados são comparáveis nos aspectos demográficos de sexo e idade, e os pacientes do ambulatório de arritmia e de doença aterosclerótica apresentam escores totais maiores quando comparados a pacientes de outros ambulatórios, nos fazendo pensar que existe maior gravidade no constructo ansiedade cardíaca nestes ambulatórios. Ao nosso conhecimento, este é o primeiro estudo brasileiro a usar o QAC em diversos grupos de pacientes cardiopatas.

Os resultados obtidos comprovam a consistência interna da escala QAC, sendo que o Alfa de Cronbach, se o item for excluído, permaneceu praticamente inalterado nos 18 itens, levando a crer que a exclusão de itens não levaria à melhora na confiabilidade da escala e os coeficientes de correlação de item total corrigidos mostraram nos 18 itens um nível aceitável. A carga fatorial de cada um dos itens nos quatro fatores também não sugeriu a exclusão de itens.

Outra proposta do presente estudo foi examinar a estrutura fatorial da escala QAC. Atualmente, existem poucos estudos nacionais que buscam verificar as dimensões desta escala e baseado nos resultados que encontramos, este instrumento mensura a ansiedade cardíaca usando quatro subescalas, sendo elas: evitação, preocupação, busca por tranquilidade e atenção. Estudos mundiais e nacionais que testaram a estrutura fatorial para suas amostras encontraram modelos com dois, três ou quatro fatores. ${ }^{9} 10,16,18,19 \mathrm{O}$ estudo conduzido por Sardinha et al. ${ }^{16}$ indicou que tanto o modelo com dois e com três fatores se adequariam à amostra brasileira, mas apontou o modelo com dois fatores como o mais interpretável. $\mathrm{O}$ nosso modelo é semelhante à solução fatorial encontrada por Marker et al. ${ }^{10}$ em sua amostra envolvendo 658 pacientes com e sem doença coronariana, onde os dados foram significativamente melhor ajustados com quatro fatores quando comparados aos modelos de um ou três fatores.

Para verificar a equivalência de parâmetros entre grupos, foram testados dois modelos para a escala, o de cargas livres para variar entre os ambulatórios e o de cargas iguais para os grupos ambulatoriais, sendo que o segundo modelo é mais restritivo do que o outro. No primeiro modelo, os parâmetros são livremente estimados para cada grupo, sendo testada apenas a plausibilidade da estrutura fatorial, ou seja, a adequação do modelo proposto para cada grupo. No segundo modelo, as cargas fatoriais são restringidas para serem iguais entre os grupos, avaliando se os itens possuem a mesma importância para os grupos. Para este estudo, foram levados em conta os índices comparativos de ajuste entre os modelos, considerandose a diferença entre o CFI de um modelo para o outro. A diferença entre o CFI ( $\delta$ CFI) não deve ser maior que 0,01 , e os resultados globais indicam a viabilidade de se restringir as cargas fatoriais para serem as mesmas nos diferentes grupos..$^{20}$ 
Na AFCMG, com o modelo com cargas iguais para os grupos ambulatoriais, não encontramos a predominância de sintomas nas subescalas avaliadas. Portanto, os itens de cada subescala não apresentaram diferenças importantes na comparação entre os grupos, levando a crer que o instrumento não está enviesado para algum grupo. Vale lembrar que se o instrumento estiver enviesado para um ou outro grupo, qualquer diferença encontrada entre os grupos pode estar relacionada não às diferenças nos sujeitos, mas sim a parâmetros não equivalentes no instrumento utilizado. ${ }^{17}$

O presente estudo comparou as médias fatoriais entre si dos cinco ambulatórios e encontrou diferenças significativas apenas na subescala evitação, onde os pacientes do ambulatório de arritmia e de doença aterosclerótica apresentam escores maiores nesta subescala quando comparados a pacientes de outros ambulatórios. $\mathrm{Na}$ prática clínica, pacientes portadores de patologias cardíacas, com maiores níveis de ansiedade, têm tendência a evitar todas as atividades associada ao risco de desenvolverem um evento cardíaco, inclusive atividades que seriam benéficas como o exercício físico leve. É importante que os cuidadores desses pacientes sejam orientados a tentar identificar um medo excessivo, que pode ser um fator limitador na execução das atividades diárias e é muito importante que o médico aborde esta questão na sua consulta.

Pesquisas longitudinais associaram a presença de ansiedade na doença coronariana a um risco elevado de morte devido à arritmia. ${ }^{21-23}$ Estudos futuros podem realizar novas análises longitudinais, para avaliar o passado, o presente e o futuro, em diferentes períodos (por exemplo, várias semanas ou anos), verificando a estabilidade das medidas de avaliação da escala QAC. Um estudo avaliou a trajetória da ansiedade

\section{REFERÊNCIAS}

1. Eifert GH. Cardiophobia: a paradigmatic behavioral model of heart-focused anxiety and non-anginal chest pain. Behav Res and Ther. 1992;30(4):329-45.

2. Zvolensky MJ, Feldner MT, Eifert GH, Vujanovic AA, Solomon SE. Cardiophobia: a critical analysis. Transcult Psychiatry. 2008;45(2):230-52

3. Eifert GH, Hodson SE, Tracey DR, Seville JL, Gunawardane K. Heart-focused anxiety, illness beliefs, and behavioral impairment: comparing healthy heart-anxious patients with cardiac and surgical inpatients. J Behav Med. 1996;19(4):385-99.

4. Eifert GH, Zvolensky MJ, Lejuez CW. Heart-focused anxiety and chest pain: a conceptual and clinical review. Clin Psychol (New York). 2000;7(4):403-17.

5. Leyro TM, Zvolensky MJ, Vujanovic AA, Johnson K, Gregor K. Perceived physical health and heart-focused anxiety among daily adult cigarette smokers: associations with affect-relevant smoking motives and outcome expectancies. Cogn Behav Ther. 2009;39(1):1123.

6. Kovacks AH, Ong L, Lin J, Chessex C. Heart-focused anxiety: the role of socioeconomic status. J Cardiopulm Rehabil. 2006;26(3):176-9. cardíaca por um ano em pacientes que sofreram infarto agudo do miocárdio e demonstrou que a qualidade de vida diminui naqueles pacientes cujos escores do QAC se mantiveram elevados. ${ }^{12}$

A verificação das propriedades psicométricas da escala QAC para uso no contexto ambulatorial e hospitalar é fundamental para o fortalecimento da discussão científica, podendo contribuir com investigações futuras ou mesmo, de acordo com o contexto, apoiar propostas de intervenções e de planejamento de políticas públicas com base em medições efetivas da ansiedade cardíaca em populações afetadas.

O presente estudo possui algumas limitações. Um ponto que pode ter influenciado os resultados é o tamanho da amostra que em algumas análises foi dividido por ambulatórios especializados. Devido ao desenho do estudo não foi possível avaliar a validade convergente e divergente da escala ou mesmo a sua estabilidade temporal. Além disso, a amostra foi coletada em ambulatórios de cardiologia especializados em apenas um hospital no estado do Ceará, o que impossibilita a generalização dos resultados para outros estados ou mesmo para atendimentos realizados por médicos generalistas.

Reconhecer a ansiedade em ambientes médicos com uma escala breve de autopreenchimento pode ajudar cardiologistas ou mesmo médicos generalistas a identificar pacientes com altos níveis de ansiedade cardíaca e o seu reconhecimento precoce pode facilitar o início de um tratamento, levando à redução no comprometimento dito disfuncional e à melhora na qualidade de vida sem necessariamente recorrer a outros profissionais de saúde ou procedimentos invasivos, especialmente em pacientes ambulatoriais considerados clinicamente estáveis.

7. Bunz M, Lenski D, Wedegartner S, Ukena C, Karbach J, Bohm $\mathrm{M}$, et al. Heart-focused anxiety in patients with chronic heart failure before implantation of an implantable cardioverter defibrillator: baseline findings of the anxiety-CHF study. Clin Res Cardiol. 2016;105(3):216-24.

8. Hamang A, Eide CE, Rokne B, Nordin K, Oyen N. General anxiety, depression, and physical health in relation to symptoms of heart-focused anxiety-a cross sectional study among patients living with the risk of serious arrhythmias and sudden cardiac death. Health Qual Life Outcomes. 2011;9:100.

9. Hoyer J, Eifert G, Einsle F, Zimmermann K, Krauss S, Knaut $\mathrm{M}$, et al. Heart-focused anxiety before and after cardiac surgery. J Psychosom Res. 2008;64(3):291-7.

10. Marker CD, Carmin CN, Ownby RL. Cardiac anxiety in people with and without coronary atherosclerosis. Depress Anxiety. 2008;25(10):824-31.

11. Rosman L, Whited A, Lampert R, Mosesso VN, Lawless C, Sears SF. Cardiac anxiety after sudden cardiac arrest: severity, predictors and clinical implications. Int J Cardiol. 2015;181:73-6.

12. van Beek $\mathrm{MH}$, Mingels $\mathrm{M}$, Voshaar RC, van Balkom AJ, Lappenschaar M, Pop G, et al. One-year follow up of cardiac anxiety 
after a myocardial infarction: a latent class analysis. J Psychosom Res. 2012;73(5):362-8.

13. Fleet RP, Dupuis G, Marchand A, Kaczorowski J, Burelle D, Arsenault A, et al. Panic disorder in coronary artery disease patients with noncardiac chest pain. J Psychosom Res. 1998;44(1):81-90.

14. Eifert GH, Thompson RN, Zvolensky MJ, Edwards K, Frazer NL, Haddad JW, et al. The cardiac anxiety questionnaire: development and preliminary validity. Behav Res Ther. 2000;38(10):1039-53.

15. Sardinha A, Nardi AE, Eifert GH. Tradução e adaptação transcultural da versão brasileira do questionário de ansiedade cardíaca. Rev psiquiatr Rio Gd Sul. 2008;30(2):139-49.

16. Sardinha A, Nardi AE, Araujo CG, Ferreira MC, Eifert GH. Validação da versão brasileira do questionário de ansiedade cardíaca. Arq Bras Cardiol. 2013;101(6):554-61.

17. Damasio BF. Contribuições da análise fatorial confirmatória multigrupo (AFCMG) na avaliação de invariância de instrumentos psicométricos. Psico-USF. 2013;18(2):211-20.

18. Dragioti E, Vitoratou S, Kaltsouda A, Tsartsalis D, Gouva M. Psychometric properties and factor structure of the greek version of the cardiac anxiety questionnaire (CAQ). Psychol Rep. 2011;109(1):77-92.

19. Fischer D, Kindermann I, Karbach J, Herzberg PY, Ukena C, Barth C, et al. Heart-focused anxiety in the general population. Clin Res Cardiology. 2012;101(2):109-16.

20. Milfont TL, Fischer R. Testing measurement invariance across groups: applications in cross-cultural research. Int J Psychol Res. 2010;3(1):111-21.

21. Kawashi I, Colditz GA, Ascherio A, Rimm EB, Giovannucci E, Stampfer MJ, et al. Prospective study of phobic anxiety and risk of coronary heart disease in men. Circulation. 1994;89(5):1992-7.

22. Albert CM, Chae CU, Rexrode KM, Manson JE, Kawashi I. Phobic anxiety and risk of coronary heart disease and sudden cardiac death among women. Circulation. 2005;111(4):480-7.

23. Watkins LL, Blumenthal JA, Davidson JR, Babyak MA, McCants $\mathrm{CB}$, Sketch MH. Phobic anxiety, depression, and risk of ventricular arrhythmias in patients with coronary heart disease. Psychosom Med. 2006;68(5):651-6.

\section{Como citar:}

Maciel PH, Silva RP, Sobrinho CR, Sá AR Junior, Pontes LM, Santos BR. Ansiedade cardíaca: uma análise em ambulatórios cardiológicos subespecializados. Rev Med UFC. 2019 jan-mar;59(1):9-15. 